\title{
Altered cortical gyrification in adults who were born very preterm and its associations with cognition and mental health
}

Chiara Papini ${ }^{1}$, Lena Palaniyappan ${ }^{2,3}$, Jasmin Kroll ${ }^{4}$, Sean Froudist-Walsh ${ }^{4,5}$, Robin M Murray ${ }^{4}$, Chiara Nosarti 6,7

1 School of Psychology, University of Nottingham, Nottingham, UK

2 Department of Psychiatry and Robarts Research Institute, University of Western Ontario, London, Ontario, Canada.

3 Lawson Health Research Institute, London, Ontario, Canada

4 Department of Psychosis Studies, Institute of Psychiatry, Psychology \& Neuroscience, King's Health Partners, King's College London, London, UK.

5 Center for Neural Science, New York University, New York, NY, USA.

6 Department of Child and Adolescent Psychiatry, Institute of Psychiatry, Psychology \& Neuroscience, King's Health Partners, King's College London, London, UK.

7 Centre for the Developing Brain, Division of Imaging Sciences \& Biomedical Engineering, King's College London, London, UK

\section{Corresponding author:}

Dr. Lena Palaniyappan MD PhD FRCPC

Robarts Research Institute, Room 1232D

1151 Richmond Street N

London, ON N6A 5B7

Email: 1palaniy@uwo.ca

Telephone: (+1) 519.931.5777

Short title: Gyrification and functional outcomes in preterm adults

Keywords: cortical folding, very preterm birth, insula, schizophrenia, neurodevelopment.

Word count: 4,000 


\begin{abstract}
Background The last trimester of pregnancy is a critical period for the establishment of cortical gyrification and altered folding patterns have been reported following very preterm birth $(<33$ weeks of gestation) in childhood and adolescence. However, research is scant on the persistence of such alterations in adulthood and their associations with cognitive and psychiatric outcomes.

Methods We studied 79 very-preterm adults and 81 age-matched full-term controls. T1-weighted images were used to measure local gyrification index (LGI), indicating the degree of folding across multiple vertices of the reconstructed cortical surface. Group and group-sex LGI differences were assessed using per-vertex adjustment for cortical thickness and overall intracranial volume. Withingroup correlations were also computed between LGI and functional outcomes, including general intelligence (IQ) and psychopathology.
\end{abstract}

$\underline{\text { Results }}$ Very preterm adults had significantly reduced LGI in extensive cortical regions encompassing the frontal, anterior temporal and occipito-parietal lobes. Alterations in lateral frontotemporal-parietal and medial occipito-parietal regions were present in both males and females, although males reported more extensive alterations. In both very preterm adults and controls, higher LGI was associated with higher IQ and lower psychopathology scores, with the spatial distribution of these associations substantially differing between the two groups.

$\underline{\text { Conclusions }}$ Very preterm adults' brains are characterized by significant and widespread local hypogyria and these abnormalities might be implicated in cognitive and psychiatric outcomes. Gyrification reflects an early developmental process and provides a fingerprint for very preterm birth. 


\section{Introduction}

Preterm birth (<37 weeks of gestation) represents over $10 \%$ of live births worldwide (1) and is associated with long-term morbidities that include psychiatric and cognitive problems $(2,3)$. The third trimester of pregnancy, when most preterm babies are born, is a critical period for brain development not only because of the dramatic increase of cortical surface and brain volume (4-6), but also for the establishment of the gyri and sulci (7). Gyrification, the folding of the cortical surface, occurs mainly in fetal life (8): the primary fissures (e.g. interhemispheric fissure) become apparent from 10-14 weeks of gestation and the sulcogyral morphology resembles the adult brain by the end of pregnancy (7). The overall ratio between buried and unburied cortex reaches adult complexity in the early postnatal period and remains relatively constant throughout development (8-11), with relatively smaller changes occurring in the first years of life (12) and beyond (13-15).

Therefore, preterm babies are at higher risk of irreversible alterations in cortical gyrification. Although sulci appear at a similar rate in babies born preterm and typically developing foetuses, the transition to the extra-uterine environment is a disruptive event for the gyrification process (16). Preterm newborns show typical but delayed gyral development at term equivalent age $(17,18)$ and exhibit less complex convolution when compared to term-born controls (19, 20). Moreover, the degree of gyrification increases with gestational age $(17,21,22)$.

Altered patterns of gyrification following very preterm birth have been reported in childhood and adolescence. Compared to term-born controls, very preterm children showed a $4 \%$ decrease of global convolution in both hemispheres, as well as regional patterns of increased gyrification (hypergyria) in the medial frontoparietal cortex and decreased gyrification (hypogyria) in the temporal cortex (23). However, mixed evidence of increased gyrification (24) and reduced sulcal surface area (25) in the temporal lobe have also been reported among preterm children. Furthermore, studies investigating the morphology of the orbitofrontal cortex in adolescence found 
reduced depth in the secondary sulci (26), but also wider sulcagyral abnormalities (27). Regional measures of gyrification in the left temporal and prefrontal cortex have been associated with language development (24) and parent-reported executive functions (27) respectively, but not with psychiatric outcomes (27). Overall, aberrant cortical folding configurations among preterm individuals appear to persist into adolescence and may be implicated in diverse and long-lasting neurobehavioral outcomes. Alterations in cortical gyrification in later life and their implications for cognitive and psychiatric outcomes are yet to be fully understood.

Regarding adult life, Hedderich et al. (28) recently investigated mean cortical curvature (29) in adults born preterm and reported extensive abnormalities across the cortical mantle, with higher magnitude of deviations associated with lower gestational age, lower birth weight and more severe medical complications at birth. This study also found associations between mean curvature and intelligence, but it did not include any psychiatric measure. Recent cross-sectional studies have revealed aberrant cortical folding in psychiatric disorders such as schizophrenia (30), bipolar disorder (31), as well as depression (32) and anxiety (33). Higher rates of mood, anxiety and psychotic disorders have been reported among preterm (3) and low birth weight individuals (34). Using the Comprehensive Assessment of At Risk Mental States (CAARMS) (35) with a dimensional approach, we recently demonstrated that adults who were born preterm experience a significant psychopathological burden and exhibit a non-specific psychiatric risk associated with lower IQ (36). Such low subclinical specificity might lead to an under-recognition of mental health problems that fall below standard diagnostic thresholds. Since cortical folding is considered a powerful indicator of early neurodevelopmental pathology (37), the identification of gyrification biomarkers of psychopathological risk following preterm birth would enable to recognize and monitor individuals who are vulnerable to develop both clinical and sub-clinical psychopathology.

In this study, we assessed a cohort of very preterm adults and a group of age-matched controls who received extensive MRI, neurocognitive and psychiatric assessment, providing 
measures of gyrification, intelligence and mental health. Specifically, to quantify cortical gyrification, we used local gyrification index (LGI) (38), a 3D version of the gold-standard gyrification index used in post-mortem studies (11) that reflects the amount of cortex buried underneath the surface of spherical cortical mantle. LGI is also relatively independent of the thickness of the cortical mantle, unlike the mean curvature (39). The aims of this study are twofold: first, to extend previous findings (28) by using LGI to assess gyrification differences across the whole brain between adults who were born very preterm and term-born individuals and relate this to general intelligence; second, to investigate whether regional variations in gyrification relate to mental health outcomes among preterm born adults. Based on previous findings, we hypothesized that preterm born participants would present different gyrification patterns, mainly hypogyria (26, 28), but also hypergyria $(23,25)$, compared to controls. Furthermore, we hypothesized positive correlations between LGI and general intelligence for very preterm individuals (28) and for controls (40). Finally, we explored the associations between LGI and psychiatric symptoms in cases and controls. 


\section{Methods and materials}

\section{Study population}

Preterm participants were recruited from a cohort of infants who were born at less than 33 weeks of gestation between 1979 and 1984 and admitted to the neonatal unit of University College Hospital, London, UK. After discharge from hospital, survivors were enrolled in a longitudinal study that involved repeated assessments in childhood (41, 42), adolescence (43) and adulthood (36, 44). Retention rate over time has been reported elsewhere $(36,45)$. Of the 152 subjects recruited for the most recent follow-up (at age 30), here we studied a sample of 83 very preterm adults.

Eighty-three aged-matched control participants were recruited from advertisements in the local community. Inclusion criteria were full-term birth (38-42 weeks of gestation) and birth weight > 2,500 grams. Exclusion criteria were birth complications (e.g. endotracheal mechanical ventilation) and neurological conditions including meningitis, head injury and cerebral infections.

The total sample consisted of participants with available MRI scan who had been assessed between February 2012 and December 2014. Participants gave full informed written consent and the study was approved by the appropriate local ethics committees, and in compliance with national legislation and the code of ethical principles for Medical Research Involving Human Subjects of the World Medical Association (Declaration of Helsinki).

\section{Socio-demographic, neuropsychological and neonatal data}

Socio-economic status (SES) was evaluated in accordance with Her Majesty's Stationary Office Occupational Classification criteria (46), using participants' occupation at current assessment and parents' jobs at birth. Categories were collapsed into two groups: high SES, which comprised managerial and professional roles, and low SES, which encompassed all the other occupations, including students and those who were currently unemployed. Hand preference was assessed using the Annett's questionnaire (47). Participants were classified as left- or right- 
dominant when consistent answers were given to all items, or mixed when inconsistent responses were reported.

Intelligence Quotient (IQ) was assessed using the Wechsler Abbreviated Scale of Intelligence (WASI) (48) that includes two verbal tasks (vocabulary and similarities) and two visuospatial tasks (block design and matrix reasoning) to provide estimates of verbal, performance and full-scale IQ expressed as standard scores $(M=100, S D=15)$. Psychiatric symptomatology was assessed with the CAARMS (35), a semi-structured interview that assess positive and negative symptoms, cognitive problems, emotional disturbance, behavioral changes, motor/physical changes and general psychopathology [see Kroll et al. (36) for further details]. The total psychopathology score, obtained as the sum of all items, was used as a measure of the non-specific psychiatric symptomatology found in our cohort (36).

For the preterm group only, neonatal variables included gestational age, birth weight, and severity of perinatal brain injury based on cranial ultrasound scan and classified as: 1) normal scan, 2) uncomplicated periventricular hemorrhage, or 3) periventricular hemorrhage and ventricular dilatation (49).

\section{MRI image acquisition and preprocessing}

Acquisition parameters of structural MRI data are described in Supplement 1. FreeSurfer v5.1.0 (http://surfer.nmr.mgh.harvard.edu/) was used to carry out the automated pre-processing including volumetric segmentation and cortical surface reconstruction $(50,51)$; these were briefly described in Supplement 1. From a total of 166 participants with available T1 sequences, six (two controls) were excluded from the analyses because of failure of the automated image processing due to substantial topological defects, skull stripping problems or poor intensity segmentation. The total sample included 79 very preterm individuals and 81 controls. 


\section{Local Gyrification Index computation}

LGI was computed using the method proposed by Schaer et al. (38) and implemented in FreeSurfer (52). This involved three steps. First, an outer hull that tightly warps the pial cortical surface was created through a morphological closing algorithm. Second, hundreds of overlapping circular regions of interest were delineated over the outer surface and matched to the corresponding patches on the pial surface. At each vertex of the hull surface, LGI was calculated as the ratio between the perimeters of the two corresponding regions, which reflected the amount of cortex buried within the sulcal folds of each region. Finally, LGI values were propagated from the outer mesh to the pial mesh in order to obtain individual cortical maps, which were subsequently used for statistical comparisons.

Visual inspection of the automatically reconstructed surfaces revealed image defects for 14 participants (three controls), mainly attributable to poor signal intensity in the occipital lobe requiring manual pial edits. A sensitivity analysis showed that results with inclusion and exclusion of those participants were unchanged (Supplemental Figure S1), therefore here we report the main analyses results including the unedited scans. Additional information about quality check and parameter choices for LGI computation are reported in Supplement 1.

\section{Statistical analyses}

Non-imaging data were analyzed using SPSS® Statistics 24.0 (53). Visual inspection plus skewness and kurtosis assessment were used to check for normal distribution of continuous variables. Parametric or non-parametric tests were used as appropriate.

To ascertain participation bias within the very preterm cohort, current study participants were compared to non-participants in terms of gestational age, birth weight, sex and neonatal US classification using independent-sample and chi-square tests respectively. 
Differences in sex, handedness and SES between very preterm individuals and controls were assessed using Pearson Chi-square test $\left(\chi^{2}\right)$, with Fisher's exact correction if required. Group differences in white matter and intracranial volume, age at assessment and neuropsychological tests were analyzed using independent-samples $t$-tests or Mann-Whitney $U$-tests as appropriate.

Between-group differences in cortical gyrification were conducted at each vertex of the cortical surface in the left and right hemispheres, using individual maps smoothed with a 10-mm full-width half-maximum Gaussian kernel. Intracranial volume was used as covariate since decreased brain volume has previously been noted in this cohort at adolescence assessment in comparison with age-matched term-born controls (54). No difference was found in our study in intracranial volume (preterm: $M[S D]=1,458.76[231.04] \mathrm{cm}^{3}$; controls: $M[S D]=1,503.53$ [205.15] $\left.\mathrm{cm}^{3} ; t_{158}=1.30, p=0.197\right)$ and its relationship with LGI did not differ between the two groups.

First, statistical analysis on LGI across the entire mantle was conducted using ANCOVA with group (very preterm and control) and sex (male and female) as between-subject factors and intracranial volume as group-centered global covariate. Main effects of group and group-sex interactions were tested. Then, group differences were tested using a per-vertex regression model where individual cortical thickness maps were entered as per-vertex regressor, while covarying for global intracranial volume. The effect of sex was tested by repeating separate analyses for male and female participants. False Discovery Rate (FDR) correction $q=0.05$ was applied to correct for multiple comparisons. Further, within-group analyses were conducted to investigate the vertex-wise correlations between LGI and: 1) full-scale IQ and 2) CAARMS measures in both very preterm and control participants, using an uncorrected significance threshold of $p=0.001$. Group differences in the relationship between LGI and functional outcomes were also tested. 


\section{Results}

\section{Sample characteristics and neuropsychological assessment}

Compared to the very preterm individuals who were not assessed at the follow-up at 30 years, the current participants had younger gestational age $\left(t_{375}=2.14, p=0.033\right)$ and lighter birth weight $\left(t_{375}=2.05, p=0.041\right)$. A higher proportion of males than females was assessed $\left(\chi_{1}^{2}=4.18\right.$, $p=0.041)$, and there were more individuals with complicated periventricular hemorrhage within the current sample $\left(\chi_{2}^{2}=6.93, p=0.031\right)$.

Table 1 displays socio-demographic and neonatal characteristics of the study sample. The two study groups did not differ in terms of sex, hand preference, parental SES at birth and participant SES at testing. Controls were younger than very preterm participants. At the neuropsychological assessment, very preterm participants had lower full-scale IQ than controls. There were no significant between-group differences in CAARMS total psychopathology. Additional measures of psychosocial functioning and mental health are reported in the Supplemental Table S1. 
Table 1. Socio-demographic and neonatal characteristics, and results of the neuropsychological assessment of the very preterm and control groups. Descriptive statistics are given in percentages or mean (standard deviation), unless otherwise stated.

\begin{tabular}{|c|c|c|c|c|c|c|}
\hline & $\begin{array}{l}\text { Preterm } \\
(n=79)\end{array}$ & $\begin{array}{l}\text { Data } \\
\text { available }(n)\end{array}$ & $\begin{array}{l}\text { Controls } \\
(n=81)\end{array}$ & $\begin{array}{l}\text { Data } \\
\text { available }(n)\end{array}$ & Test statistic & $p$ value \\
\hline Sex (male/female) & $61 / 39$ & 79 & $48 / 52$ & 81 & $\chi_{1}^{2}=2.56$ & 0.109 \\
\hline Age at assessment (in years) ${ }^{a}$ & $30.24(3.95)$ & 78 & $29.29(5.02)$ & 79 & $U=2,439.00$ & 0.024 \\
\hline Handedness (right/left/mixed) & $83 / 10 / 7$ & 60 & $88 / 7 / 5$ & 57 & Fisher's exact $\chi_{1}^{2}=0.54$ & 0.856 \\
\hline Subject SES at testing (low/high) & $53 / 47$ & 78 & $51 / 49$ & 80 & $\chi_{1}^{2}=0.03$ & 0.869 \\
\hline Parental SES at birth (low/high) & $49 / 51$ & 73 & $35 / 65$ & 65 & $\chi_{1}^{2}=2.73$ & 0.099 \\
\hline \multicolumn{7}{|l|}{ Neonatal characteristics } \\
\hline Birth weight (in grams) & $1280.82(339.88)$ & 78 & $\mathrm{n} / \mathrm{a}$ & & - & - \\
\hline Gestational age (in weeks) & $29.10(2.21)$ & 78 & $\mathrm{n} / \mathrm{a}$ & & - & - \\
\hline US classification (no-PVH/PVH/PVH+DIL) & $47 / 23 / 30$ & 79 & $\mathrm{n} / \mathrm{a}$ & & - & - \\
\hline \multicolumn{7}{|l|}{ Neuropsychological assessment } \\
\hline Full-scale IQ $^{\text {a }}$ & $107(17)$ & 68 & $115(14)$ & 64 & $U=1,469.00$ & 0.001 \\
\hline CAARMS total psychopathology ${ }^{a}$ & $7(14)$ & 65 & $4(10)$ & 68 & $U=1,847.50$ & 0.101 \\
\hline
\end{tabular}

Note: missing data were mainly due to logistic problems at the beginning of the project and incomplete items preventing the computation of composite scores.

${ }^{a}$ Median (interquartile range).

CAARMS = Comprehensive Assessment of At-Risk Mental States. IQ = Intelligence Quotient. SES = socio-economic status.

Ultrasound Scan (US) classification: no-PVH = normal neonatal cranial ultrasound; PVH = uncomplicated periventricular hemorrhage; PVH + DIL = periventricular hemorrhage with ventricular dilatation. 


\section{Gyrification differences between very preterm and control participants}

Figure 1A shows the results of the ANCOVA testing for differences in LGI between very preterm individuals and controls, accounting for sex, while covarying for intracranial volume. Compared to term-born controls, very preterm participants showed reduced gyrification in two clusters, one in the left hemisphere $\left(\right.$ size $\left.=61,036 \mathrm{~mm}^{2}\right)$ and one in the right hemisphere $($ size $=$ $\left.56,036 \mathrm{~mm}^{2}\right)$, both with peak in the insula $(p<.001)$. The clusters extended to large portions of prefrontal, anterior temporal, medial and dorsal parietal, and occipital lobes bilaterally as well as in the left superior frontal gyrus. Moreover, preterm individuals showed increased gyrification in a small medial portion of the right temporal pole ( $\left.\operatorname{size}=76 \mathrm{~mm}^{2}, p<.01\right)$. There was no significant interaction between sex and group $(p>0.05)$.

Figure 1B and Table 2 show the results of the per-vertex regression model assessing group differences in LGI using intracranial volume and cortical thickness at each vertex of the surface as confounders. Very preterm participants showed significantly reduced cortical folding bilaterally in the insula, middle frontal and orbitofrontal gyri, anterior temporal lobe, prefrontal cortex and extended parts of the medial occipito-parietal cortices compared to term-born controls (see also Supplemental Figure S3). Separate analyses for sex yielded a similar pattern of hypogyria involving the insula, anterior temporal and medial parietal-occipital areas, whereas reduced folding in the frontal and lateral occipito-parietal lobes appeared more prominent in males than females. Conversely, the hypergyric region in the temporal lobe did not remain significant for either sex.

Overlapping results were obtained when intracranial volume was not included as a global covariate, with significant group effects found in nearly all the same regions (Supplemental Figure S2). 


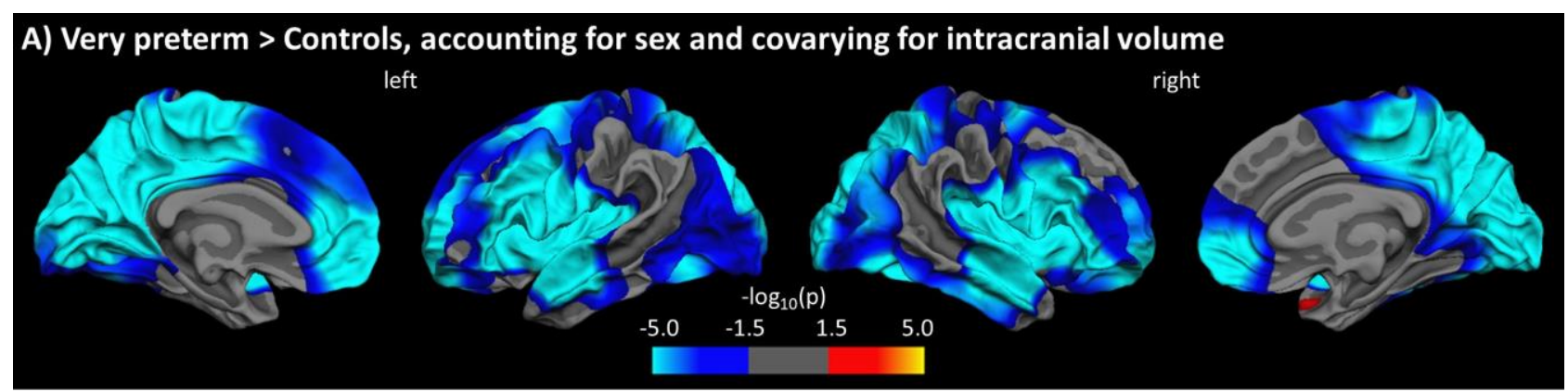

B) Very preterm > Controls, covarying for intracranial volume and vertex-wise cortical thickness
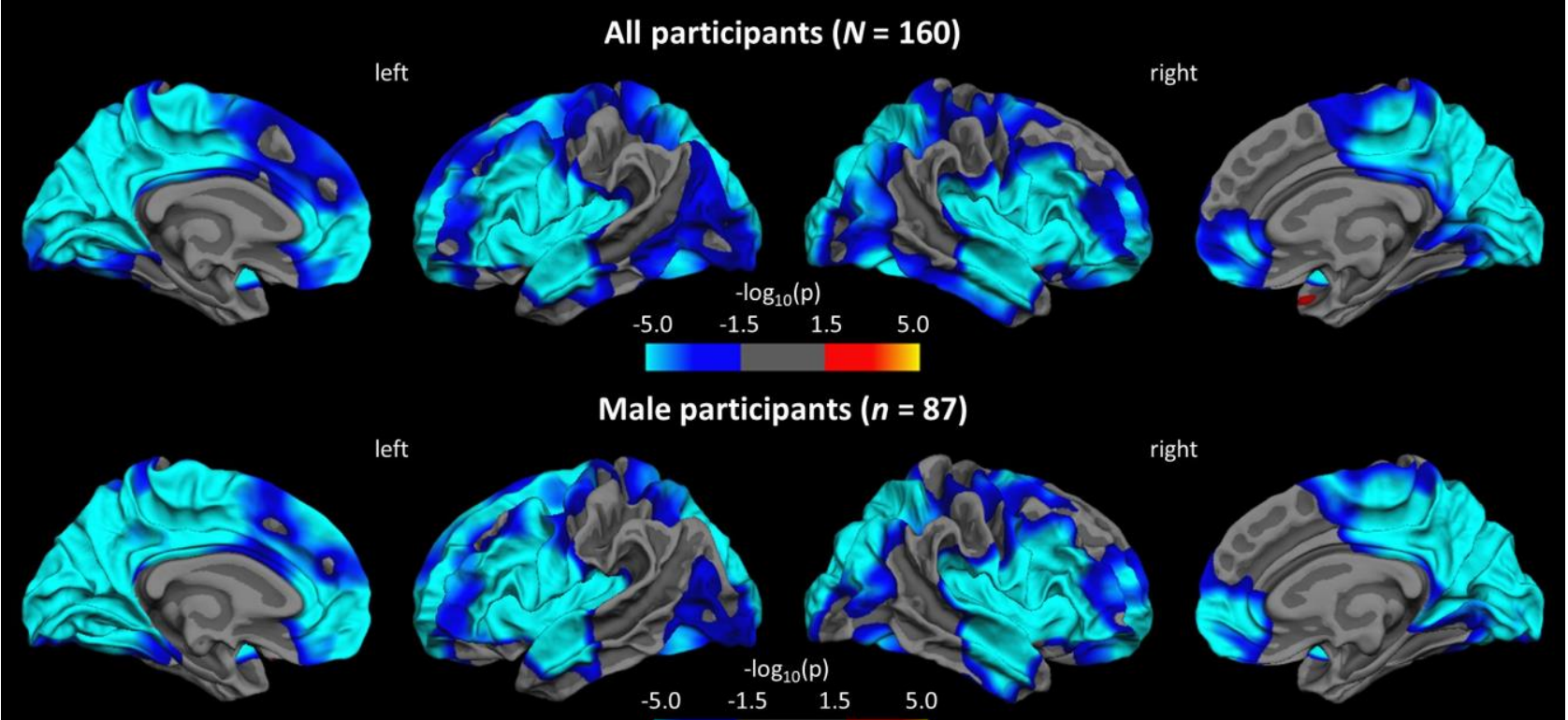

left

Male participants $(n=87)$
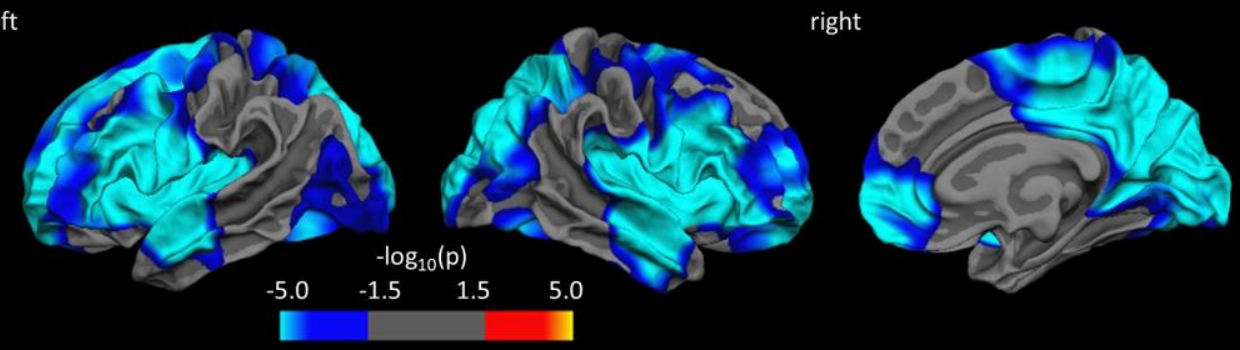

Female participants $(n=73)$

left
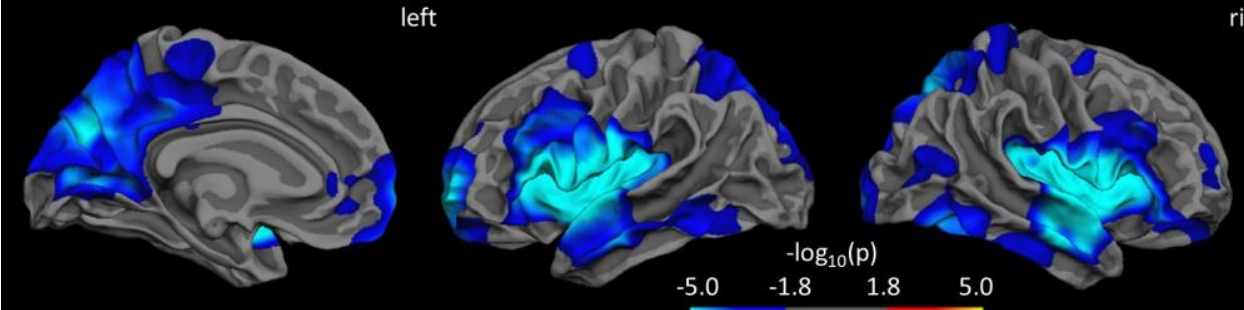

right

Figure 1. Results from general linear models testing for group differences in LGI: A) accounting for sex, with intracranial volume as global covariate; B) covarying for intracranial volume and vertex-wise cortical thickness for all (top row), male (middle row), and female (bottom row) participants. Statistical maps are displayed on the white surface, with FDR-corrected level of significance at 0.05 . 
Table 2. Significant clusters showing group differences in LGI while covarying for intracranial volume and adjusting for vertex-wise cortical thickness (FDR correction $q=0.05$ ).

\begin{tabular}{|c|c|c|c|c|c|c|}
\hline \multirow[t]{2}{*}{ Subjects } & \multirow[t]{2}{*}{ Cortical region $(\mathrm{H})$} & \multirow[t]{2}{*}{$\begin{array}{l}\text { Cluster size, } \\
\mathrm{mm}^{2}\end{array}$} & \multirow[t]{2}{*}{$p$ value } & \multicolumn{3}{|c|}{$\begin{array}{c}\text { Talaraich coordinates } \\
\text { of the max }\end{array}$} \\
\hline & & & & $\mathrm{x}$ & $\mathrm{y}$ & $\mathrm{z}$ \\
\hline \multirow[t]{3}{*}{ Total sample } & Precentral (L) & 59,410 & $<.001$ & -39 & 0 & 15 \\
\hline & Precentral (R) & 54,116 & $<.001$ & 53 & -2 & 10 \\
\hline & Superior temporal (R) & 49 & $<.05$ & 40 & 11 & -26 \\
\hline \multirow[t]{5}{*}{ Males } & Superior temporal (L) & 55,708 & $<.001$ & -43 & -20 & -6 \\
\hline & Lateral orbitofrontal (L) & 23 & $<.05$ & -26 & 18 & -19 \\
\hline & Superior temporal (R) & 23,094 & $<.001$ & 52 & -10 & -1 \\
\hline & Precuneus (R) & 29,031 & $<.001$ & 10 & -66 & 39 \\
\hline & Caudal middle frontal (R) & 3 & $<.05$ & 29 & 12 & 43 \\
\hline \multirow[t]{14}{*}{ Females } & Precentral (L) & 14,684 & $<.001$ & -48 & -1 & 8 \\
\hline & Rostral middle frontal (L) & 2,471 & $<.001$ & -16 & 61 & -5 \\
\hline & Precuneus (L) & 12,472 & $<.001$ & -19 & -69 & 30 \\
\hline & Superior frontal (L) & 292 & $<.01$ & -19 & 5 & 56 \\
\hline & Medial orbitofrontal (L) & 110 & $<.01$ & -12 & 40 & -7 \\
\hline & Rostral anterior cingulate $(\mathrm{L})$ & 62 & $<.01$ & -6 & 35 & 3 \\
\hline & Inferior parietal (L) & 73 & $<.05$ & -43 & -56 & 41 \\
\hline & Posterior cingulate (L) & 16 & $<.05$ & -8 & -24 & 29 \\
\hline & Precentral (L) & 19 & $<.05$ & -21 & -22 & 52 \\
\hline & Insula (R) & 28,447 & $<.001$ & 37 & -3 & 16 \\
\hline & Superior frontal (R) & 153 & $<.01$ & 22 & 1 & 53 \\
\hline & Inferior parietal (R) & 726 & $<.01$ & 44 & -71 & 18 \\
\hline & Rostral middle frontal (R) & 279 & $<.01$ & 22 & 55 & 15 \\
\hline & Rostral middle frontal (R) & 3 & $<.05$ & 39 & 42 & -1 \\
\hline
\end{tabular}

$\mathrm{H}=$ hemisphere; $\mathrm{L}=$ left; $\mathrm{R}=$ right. 


\section{Per-vertex correlations between cortical gyrification and full-scale IQ}

Positive vertex-wise associations were found between LGI and full-scale IQ in separategroup analyses for preterm and control participants. In very preterm participants, associations between LGI and full-scale IQ were found in the left fusiform and lateral orbitofrontal cortex as well as in the right superior parietal gyrus (Figure 2A, Table 3). In control participants associations between LGI and full-scale IQ were found mainly in the right inferior parietal lobe, postcentral gyrus, lateral orbitofrontal and caudal middle frontal cortex, but also in the left pars orbitalis (Figure 2B; Table 3). There was no group-by-IQ interaction on LGI $(p>0.05)$.

\section{Per-vertex correlations between cortical gyrification and CAARMS total psychopathology}

Vertex-wise correlations between LGI and CAARMS total psychopathology scores were conducted after removal of two participants (one very preterm and one control) who were identified as outliers with unusually high CAARMS scores. Negative correlations were found throughout, but these involved different brain areas in each group. Very preterm participants displayed significant associations between LGI and CAARMS total psychopathology scores in the left precentral, bilateral postcentral, right paracentral regions as well as in the right insula (Figure 2C, Table 3). Control participants showed only a significant association in the right supramarginal gyrus (Figure 2D, Table 3). There was no group-by-CAARMS interaction on LGI $(p>0.05)$. 


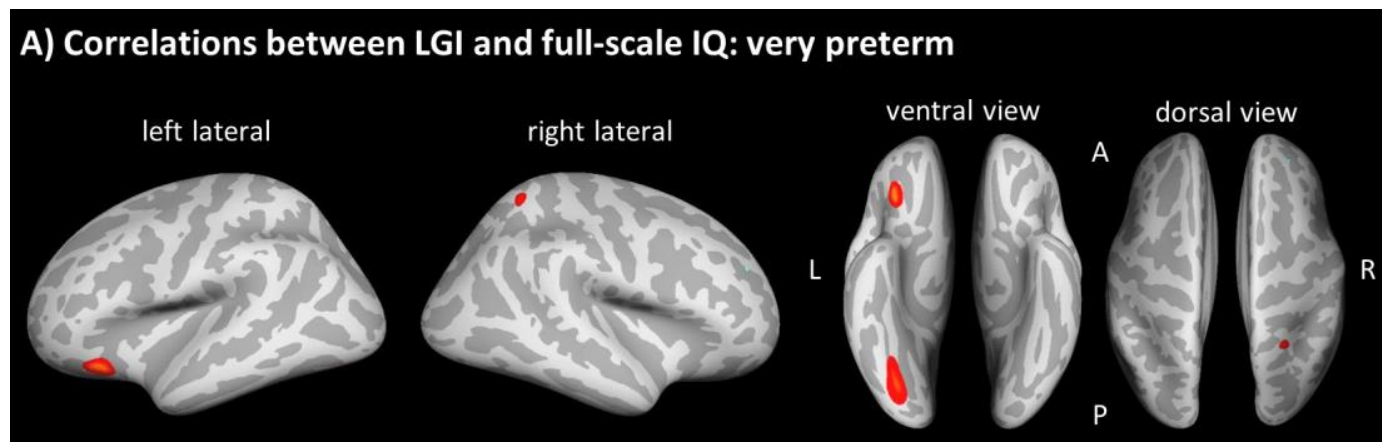

B) Correlations between LGI and full-scale IQ: control

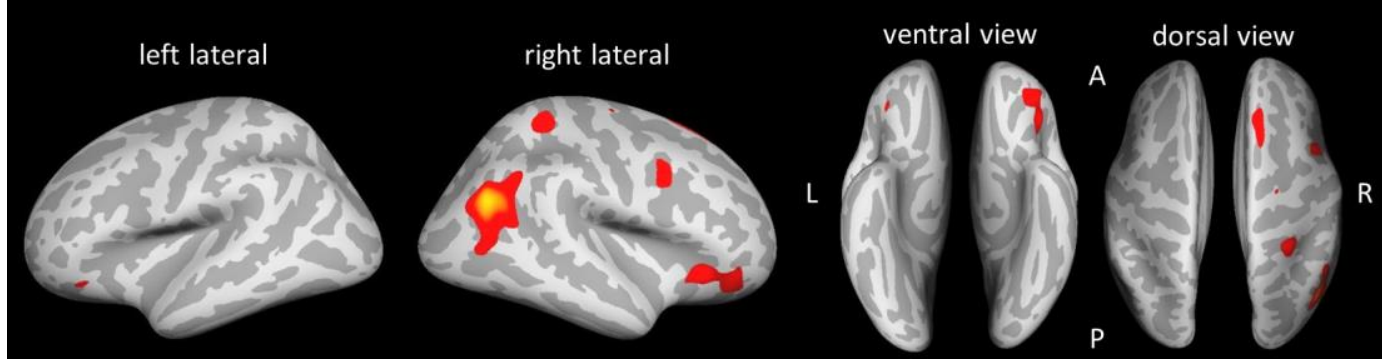

C) Correlations between LGI and CAARMS total psychopathology: very preterm

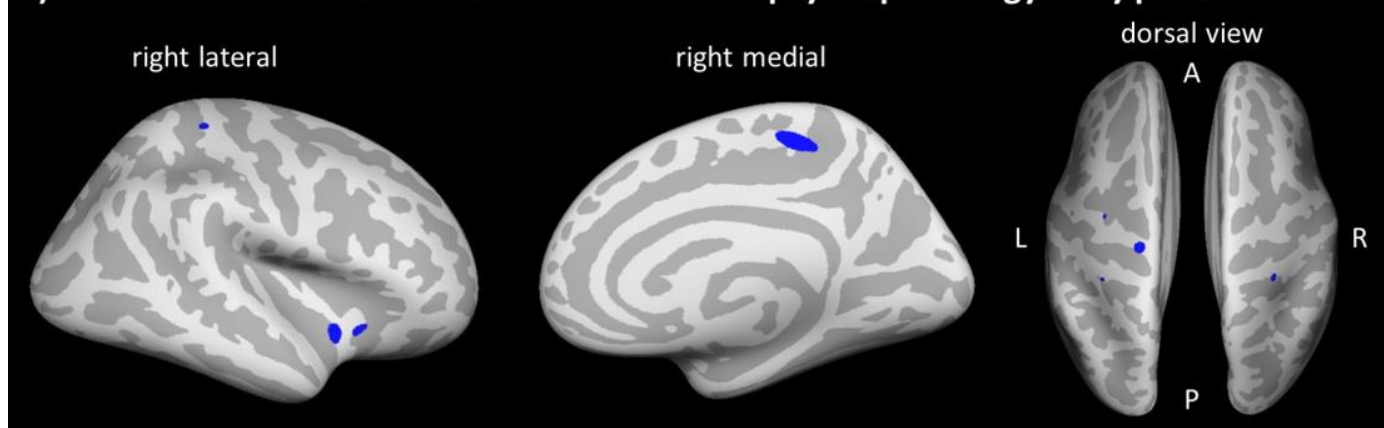

D) Correlations between LGI and CAARMS total psychopathology: control

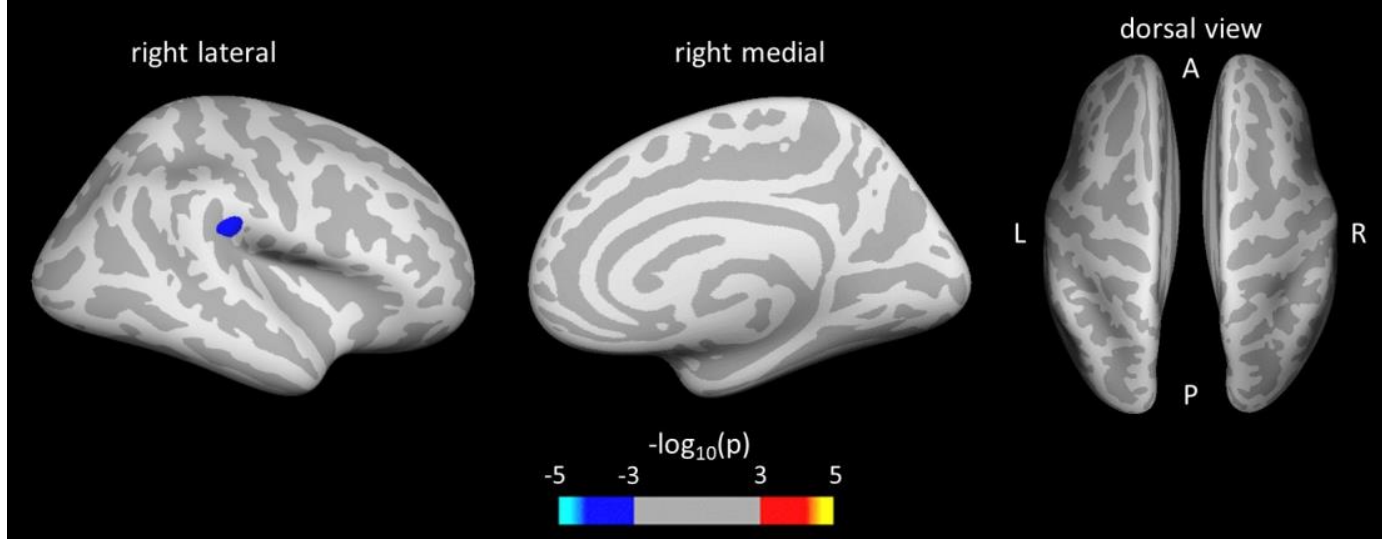

Figure 2. Vertex-wise associations of LGI with full-scale IQ and CAARMS total psychopathology for very preterm and control participants. Statistical maps are displayed on the inflated surface, with uncorrected level of significance $p=0.001$. LGI $=$ local gyrification index. 
Table 3. Significant associations of LGI with full-scale IQ (positive) and CAARMS total psychopathology (negative) for very preterm and control participants (all uncorrected $p<.001$ ).

\begin{tabular}{|c|c|c|c|c|c|c|}
\hline \multirow[t]{2}{*}{ Measure } & \multirow[t]{2}{*}{ Subgroups } & \multirow[t]{2}{*}{ Cortical region $(\mathrm{H})$} & \multirow[t]{2}{*}{$\begin{array}{l}\text { Cluster size, } \\
\mathrm{mm}^{2}\end{array}$} & \multicolumn{3}{|c|}{$\begin{array}{c}\text { Talaraich coordinates } \\
\text { of the max }\end{array}$} \\
\hline & & & & $\mathrm{x}$ & $\mathrm{y}$ & $\mathrm{z}$ \\
\hline \multirow[t]{10}{*}{ Full-scale IQ } & \multirow{3}{*}{ Very preterm } & Lateral orbitofrontal (L) & 341 & -35 & 22 & -16 \\
\hline & & Fusiform (L) & 614 & -29 & -74 & -6 \\
\hline & & Superior parietal (R) & 94 & 31 & -48 & 61 \\
\hline & \multirow[t]{7}{*}{ Controls } & Pars orbitalis (L) & 45 & -40 & 29 & -13 \\
\hline & & Inferior parietal $(\mathrm{R})$ & 1,486 & 45 & -61 & 26 \\
\hline & & Postcentral (R) & 247 & 25 & -36 & 50 \\
\hline & & Superior frontal (R) & 303 & 17 & 20 & 52 \\
\hline & & Lateral orbitofrontal (R) & 676 & 36 & 29 & -5 \\
\hline & & Caudal middle frontal (R) & 235 & 36 & 7 & 34 \\
\hline & & Precentral (R) & 24 & 25 & -10 & 60 \\
\hline \multirow[t]{8}{*}{ CAARMS } & \multirow[t]{7}{*}{ Very preterm } & Precentral (L) & 48 & -17 & -19 & 67 \\
\hline & & Postcentral (L) & 14 & -34 & -31 & 57 \\
\hline & & Precentral (L) & 10 & -32 & -9 & 49 \\
\hline & & Paracentral (R) & 123 & 10 & -29 & 49 \\
\hline & & Postcentral (R) & 26 & 30 & -31 & 58 \\
\hline & & Insula (R) & 69 & 37 & 0 & -6 \\
\hline & & Insula (R) & 31 & 31 & 18 & -4 \\
\hline & Control & Supramarginal (R) & 184 & 49 & -25 & 26 \\
\hline
\end{tabular}

$\mathrm{H}=$ hemisphere; $\mathrm{L}=$ left; $\mathrm{R}=$ right. 


\section{Discussion}

Using a three-dimensional measure of gyrification, this study showed altered gyrification in a large portion of the cortical mantle in adults who were born very preterm compared to agematched term-born controls. Specifically, our results confirm the primary hypothesis of mostly reduced cortical folding, in line with previous evidence involving the orbitofrontal (26), insular and superior temporal lobes $(20,23)$ in preterm children and adolescents. Our observations partially confirm the results by Hedderich et al. (28), who reported widespread curvature reduction in very preterm/low-birth-weight adults extended to the parietal and temporal lobes, but not to the medial and lateral superior frontal cortex. Differences between the two studies might arise from the different 3D surface-based proxies of folding complexity.

In addition, the current study found a small hypergyria in the temporal lobe, but this pattern should be interpreted cautiously due to its inconsistency, as demonstrated in the sex-separate comparisons. Kesler et al. (24) demonstrated increased temporal lobe folding using a global 2D gyrification index in very preterm children, but other studies reported reduced folding complexity in this lobe $(18,23)$. The current study with a regional proxy suggests a specific vulnerability to both hypo- and hyper-gyrification of different temporal regions, although the underpinning mechanism remains to be elucidated (23). In line with the previous literature, our results altogether demonstrate significant gyrification abnormalities (both increased and decreased) in very preterm subjects as compared to term-born controls.

Sex did not show significant effects on gyrification, possibly because adjustment for brain volume accounted for folding differences between male and female adults (55). Nonetheless, sexspecific comparisons suggested that folding alterations were more prominent in male adults, in line with extensive research showing higher vulnerability for male preterm infants to neurodevelopmental morbidities (56-58) as well as structural (59) and functional (60) brain 
alterations. In addition, preterm girls have a more "compact" brain, with higher degree of cortical folding but smaller brain volume (61), which may have important implications for later outcomes and psychopathology (62). Furthermore, vertex-wise adjustment for cortical thickness, which is an indicator of brain maturation during childhood and adolescence (63), did not account for differences in adulthood on gyrification, which instead reflects early brain development during fetal life (37). Therefore, reduced gyrification detected in our adult sample could be interpreted as the result of an early alteration to the folding process in perinatal life that persists beyond cortical maturation programmed to occur later in life.

Using a whole brain approach, we found the strongest gyrification reduction in the very preterm sample bilaterally in the insula, a deeply buried region characterized by elevated convolution complexity across lifespan $(12,64)$. The insula, the first lobe to differentiate in fetal life, starts sulcation/gyrification at 16 weeks of gestation $(4,65)$ and undergoes critical development between 21 and 32 weeks (66), making it particularly vulnerable to alterations following very preterm birth. Nonetheless, the hypogyria consistently extended beyond the insula to the anterior temporal lobe, inferior frontal lobe, orbitofrontal cortex, and medial occipito-parietal regions, and its robustness was demonstrated in both male and female participants. Our observation shows a striking overlap with the results of a whole-brain study in individuals with adult-onset schizophrenia reporting reduced gyrification in the insular region with extensions to the inferior frontal gyrus and superior temporal gyrus (30). Both increased and decreased gyrification in these areas were also found in another sample of adolescents with schizophrenia (67), as well as in other psychotic and nonpsychotic conditions, such as bipolar disorder and depression (32), which are more prevalent in the preterm population (3). Therefore, our findings imply that aberrant gyrification seen in individuals with various psychiatric disorders could at least partly be explained by neurodevelopmental disruptions occurring very early in life. 
Gyrification showed a positive linear relationship with full-scale IQ in both very preterm and control participants, especially in the left orbitofrontal and right superior parietal regions. A distinctive association for the preterm group was found in the left fusiform. For the control group, unique associations involved the middle and inferior frontal lobe as well as the temporo-parietal junction in the right hemisphere. These findings corroborate evidence of a wide fronto-parietal network sustaining the association between WASI full-scale IQ and LGI in a large cohort of healthy adults (40). Notably, in the current study the significant group difference in full-scale IQ probably reflects not so much an impairment of very preterm participants but rather an overperformance of control participants who tend to score $+1 \mathrm{SD}$ above the test mean, arguably because many were university students (Supplement, Table S1). Therefore, it is plausible that the network containment and the reduced involvement of associative cortices might be implicated in the lowered IQ found in very preterm participants in comparison to term-born controls.

Finally, exploratory analyses showed the same direction of negative associations between local gyrification and psychopathology scores in both very preterm and control groups, but different spatial distribution across the whole brain. In controls, one significant association was found only in the right supramarginal gyrus, an area specifically implicated in empathic judgements (68) and social cognition through the frontoparietal mirror system (69). Instead, in the very preterm group the association involved the central and paracentral lobules, corresponding to the primary sensorymotor cortices (70), and the right insula, specialized in interoception, multimodal and somatosensorial processing $(71,72)$ and emotional awareness (73). The CAARMS, a tool specifically designated to recognize the subclinical symptomatology of a first psychotic episode (35), includes questions about perception and body-sensation abnormalities as well as emotionalaffective dysfunction. Therefore, while higher gyrification is associated with better adult mental health in both preterm and term-born individuals, the regional specificity of the relationships suggests that the link between early gyrification process and later psychiatric problems is 
qualitatively different in the two groups because of the different neural substrates. The current study is the first to show significant associations between gyrification and psychiatric outcomes in preterm individuals, which were not examined $(24,26,28)$ or demonstrated (27) previously. While the paucity of research and methodological differences might explain this novel finding, it is also possible that such associations arise only later in adult life, in continuity with childhood psychopathology (74) even when considering subclinical symptoms (75). The initial delay of the folding process following very preterm birth (17-20) might contribute to the faster LGI decline seen in various psychiatric diagnoses (64). However, more research is necessary in future to elucidate the nature of the relationship between morphological features and dimensional functional outcomes, i.e. to establish concurrent and predictive associations throughout life and in relation to sex differences (62).

Several limitations should be considered when evaluating this research. Attrition analysis suggests that we considered a high-risk sample because all perinatal risk factors were not favourable in our preterm group in comparison to the non-returning cohort, unlike what commonly occur in longitudinal studies (76). However, the neuroimaging nature of this study might have involved high-functioning participants who were able to undergo an MRI scan (77), as suggested also by the similar psychopathology levels reported in preterm and control participants. Furthermore, our preterm cohort born three decades ago may not be representative of more recent preterm cohorts who receive novel neuroprotective interventions (78), but long-term sequelae seem to persist in spite of advancements in neonatal care (79). Our control group had a slightly younger mean age at assessment compared to the preterm group; data plotting also showed that controls comprised a few older individuals. However, since LGI decreases with age in healthy individuals $(14,64)$, the presence of these participants is expected to have attenuated group differences in our study. Finally, another limitation regards the use of the CAARMS, which is designed for individuals at ultra-high risk of psychosis. Future research should employ a diagnostic interview 
that is for use with the general population and examines various psychiatric disorders (80), or selfreported psychopathology assessment, that might be sensitive to subclinical symptoms (81).

To conclude, the current study found widespread reduced cortical gyrification in very preterm adults compared to term-born controls, particularly in the middle and inferior frontal, superior temporal and medial occipito-parietal regions. It also described specific regional associations of local gyrification with cognitive ability and mental health outcomes in adulthood. Overall, this study demonstrates that gyrification measured in adult life provides a fingerprint for very preterm birth, with wide aberrations persisting in adult life and possibly implicated in functional outcomes. 


\section{Acknowledgments}

The study was funded by a Medical Research Council, UK research grant (ref. MR/K004867/1) to CN. LP acknowledges support from the Tanna Schulich Chair of Neuroscience and Mental Health and the Opportunities Fund of the Academic Health Sciences Centre Alternative Funding Plan of the Academic Medical Organization of Southwestern Ontario (AMOSO).

We thank our study participants for their continuing help. We also thank the National Institute for Health Research (NIHR) Biomedical Research Center at South London and Maudsley NHS Foundation Trust and King's College London for supporting the neuroimaging facilities used in our study. 


\section{References}

1. Chawanpaiboon S, Vogel JP, Moller AB, Lumbiganon P, Petzold M, Hogan D, et al. (2019): Global, regional, and national estimates of levels of preterm birth in 2014: a systematic review and modelling analysis. Lancet Glob Heal. 7: e37-e46.

2. Nosarti C, Nam KW, Walshe M, Murray RM, Cuddy M, Rifkin L, Allin MPG (2014): Preterm birth and structural brain alterations in early adulthood. NeuroImage Clin. 6: 180-191.

3. Nosarti C, Reichenberg A, Murray RM, Cnattingius S, Lambe MP, Yin L, et al. (2012): Preterm birth and psychiatric disorders in young adult life. Arch Gen Psychiatry. 69: E1-E8.

4. Clouchoux C, Kudelski D, Gholipour A, Warfield SK, Viseur S, Bouyssi-Kobar M, et al. (2012): Quantitative in vivo MRI measurement of cortical development in the fetus. Brain Struct Funct. 217: 127-139.

5. Habas PAA, Scott JAA, Roosta A, Rajagopalan V, Kim K, Rousseau F, et al. (2012): Early folding patterns and asymmetries of the normal human brain detected from in utero MRI. Cereb Cortex. 22: 13-25.

6. Wright R, Kyriakopoulou V, Ledig C, Rutherford MA, Hajnal J V, Rueckert D, Aljabar P (2014): Automatic quantification of normal cortical folding patterns from fetal brain MRI. Neuroimage. 91: 21-32.

7. Chi JGG, Dooling ECC, Gilles FHH (1977): Gyral development of the human brain. Ann Neurol. 1: 86-93.

8. White T, Su S, Schmidt M, Kao C-Y, Sapiro G (2010): The development of gyrification in childhood and adolescence. Brain Cogn. 72: 36-45.

9. Zilles K, Schleicher A, Langemann C, Amunts K, Morosan P, Palomero-Gallagher N, et al. (1997): Quantitative analysis of sulci in the human cerebral cortex: development, regional heterogeneity, gender difference, asymmetry, intersubject variability and cortical architecture. Hum Brain Mapp. 5: 218-221.

10. Armstrong E, Schleicher A, Omran H, Curtis M, Zilles K (1995): The ontogeny of human gyrification. Cereb Cortex. 5: 56-63.

11. Zilles K, Armstrong E, Schleicher A, Kretschmann H-J (1988): The human pattern of gyrification in the cerebral cortex. Anat Embryol (Berl). 179: 173-179.

12. Li G, Wang L, Shi F, Lyall AE, Lin W, Gilmore JH, Shen D (2014): Mapping longitudinal development of local cortical gyrification in infants from birth to 2 years of age. $J$ Neurosci. 34: 4228-4238.

13. Raznahan A, Shaw P, Lalonde F, Stockman M, Wallace GL, Greenstein D, et al. (2011): How does your cortex grow? J Neurosci. 31: 7174-7177.

14. Hogstrom LJ, Westlye LT, Walhovd KB, Fjell AM (2013): The structure of the cerebral cortex across adult life: age-related patterns of surface area, thickness, and gyrification. Cereb Cortex. 23: 2521-2530.

15. Klein D, Rotarska-Jagiela A, Genc E, Sritharan S, Mohr H, Roux F, et al. (2014): Adolescent brain maturation and cortical folding: evidence for reductions in gyrification. PLoS One. 9: e84914.

16. Lefèvre J, Germanaud D, Dubois J, Rousseau F, De MacEdo Santos I, Angleys H, et al. (2016): Are developmental trajectories of cortical folding comparable between cross-sectional datasets of fetuses and preterm newborns? Cereb Cortex. 26: 3023-3035. 
17. Dubois J, Benders M, Cachia A, Lazeyras F, Ha-Vinh Leuchter R, Sizonenko S V, et al. (2008): Mapping the early cortical folding process in the preterm newborn brain. Cereb Cortex. 18: 1444-1454.

18. Battin MR, Maalouf EF, Counsell SJ, Herlihy AH, Rutherford MA, Azzopardi D, Edwards AD (1998): Magnetic resonance imaging of the brain in preterm infants: visualization of the germinal matrix, early myelination, and cortical folding. Pediatr Neuroradiol. 101: 957-962.

19. Ajayi-Obe M, Saeed N, Cowan FM, Rutherford MA, Edwards AD (2000): Reduced development of cerebral cortex in extremely preterm infants. Lancet. 356: 1162-1163.

20. Engelhardt E, Inder TE, Alexopoulos D, Dierker DL, Hill J, Van Essen D, Neil JJ (2015): Regional impairments of cortical folding in premature infants. Ann Neurol. 77: 154-162.

21. Melbourne A, Kendall GSS, Cardoso MJJ, Gunny R, Robertson NJJ, Marlow N, Ourselin S (2014): Preterm birth affects the developmental synergy between cortical folding and cortical connectivity observed on multimodal MRI. Neuroimage. 89: 23-34.

22. Benders MJJ, Palmu K, Menache C, Borradori-Tolsa C, Lazeyras F, Sizonenko S, et al. (2015): Early brain activity relates to subsequent brain growth in premature infants. Cereb Cortex. 25: 3014-3024.

23. Zhang Y, Inder TE, Neil JJ, Dierker DL, Alexopoulos D, Anderson PJ, Van Essen DC (2015): Cortical structural abnormalities in very preterm children at 7 years of age. Neuroimage. 109: 469-479.

24. Kesler SR, Vohr B, Schneider KC, Katz KH, Makuch RW, Reiss AL, Ment LR (2006): Increased temporal lobe gyrification in preterm children. Neuropsychologia. 44: 445-453.

25. Zubiaurre-Elorza L, Soria-Pastor S, Junqué C, Vendrell P, Padilla N, Rametti G, et al. (2009): Magnetic resonance imaging study of cerebral sulci in low-risk preterm children. Int $J$ Dev Neurosci. 27: 559-565.

26. Giménez M, Junqué C, Vendrell P, Narberhaus A, Bargalló N, Botet F, Mercader JM (2006): Abnormal orbitofrontal development due to prematurity. Neurology. 67: 1818-1822.

27. Ganella EP, Burnett A, Cheong J, Thompson D, Roberts G, Wood S, et al. (2015): Abnormalities in orbitofrontal cortex gyrification and mental health outcomes in adolescents born extremely preterm and/or at an extremely low birth weight. Hum Brain Mapp. 36: 11381150.

28. Hedderich DM, Bäuml JG, Berndt MT, Menegaux A, Scheef L, Daamen M, et al. (2019): Aberrant gyrification contributes to the link between gestational age and adult IQ after premature birth. Brain. 142: 1255-1269.

29. Luders E, Thompson PM, Narr KL, Toga AW, Jancke L, Gaser C (2006): A curvature-based approach to estimate local gyrification on the cortical surface. Neuroimage. 29: 1224-1230.

30. Palaniyappan L, Liddle P (2012): Aberrant cortical gyrification in schizophrenia: a surfacebased morphometry study. J Psychiatry Neurosci. 37: 399-406.

31. Mirakhur A, Moorhead TWJ, Stanfield AC, McKirdy J, Sussmann JED, Hall J, et al. (2009): Changes in gyrification over 4 years in bipolar disorder and their association with the brainderived neurotrophic factor valine(66) methionine variant. Biol Psychiatry. 66: 293-297.

32. Zhang Y, Yu C, Zhou Y, Li K, Li C, Jiang T (2009): Decreased gyrification in major depressive disorder. Neuroreport. 20: 378-380.

33. Molent C, Maggioni E, Cecchetto F, Garzitto M, Piccin S, Bonivento C, et al. (2018): Reduced cortical thickness and increased gyrification in generalized anxiety disorder: a 3 T MRI study. 
Psychol Med. 48: 2001-2010.

34. Mathewson KJ, Chow CHT, Dobson KG, Pope EI, Schmidt LA, Van Lieshout RJ (2017): Mental health of extremely low birth weight survivors: a systematic review and meta-analysis. Psychol Bull. 143: 347-383.

35. Yung AR, Yuen HP, McGorry PD, Phillips LJ, Kelly D, Dell'Olio M, et al. (2005): Mapping the onset of psychosis: the Comprehensive Assessment of At-Risk Mental States. Aust $N Z J$ Psychiatry. 39: 964-71.

36. Kroll J, Froudist-Walsh S, Brittain PJ, Tseng C-EJ, Karolis V, Murray RM, Nosarti C (2018): A dimensional approach to assessing psychiatric risk in adults born very preterm. Psychol Med. 48: $1738-1744$.

37. Schaer M, Eliez S (2009): Contribution of structural brain imaging to our brain development. Eur Psychiatry Rev. 2: 13-16.

38. Schaer M, Cuadra MB, Tamarit L, Lazeyras F, Eliez S, Thiran J-P (2008): A surface-based approach to quantify local cortical gyrification. IEEE Trans Med Imaging. 27: 161-170.

39. Forde NJ, Ronan L, Zwiers MP, Schweren LJS, Alexander-Bloch AF, Franke B, et al. (2017): Healthy cortical development through adolescence and early adulthood. Brain Struct Funct. 222: 3653-3663.

40. Gregory MD, Kippenhan JS, Dickinson D, Carrasco J, Mattay VS, Weinberger DR, Berman KF (2016): Regional variations in brain gyrification are associated with general cognitive ability in humans. Curr Biol. 26: 1301-1305.

41. Roth SC, Baudin J, Pezzani-Goldsmith M, Townsend J, Reynolds EO, Stewart AL (1994): Relation between neurodevelopmental status of very preterm infants at one and eight years. Dev Med Child Neurol. 36: 1049-1062.

42. Stewart AL, Costello AM, Hamilton PA, Baudin J, Townsend J, Bradford BC, Reynolds EO (1989): Relationship between neurodevelopmental status of very preterm infants at one and four years. Dev Med Child Neurol. 31: 756-765.

43. Nam KW, Castellanos N, Simmons A, Froudist-Walsh S, Allin MP, Walshe M, et al. (2015): Alterations in cortical thickness development in preterm-born individuals: implications for high-order cognitive functions. Neuroimage. 115: 64-75.

44. Kroll J, Karolis V, Brittain PJ, Tseng C-EJ, Froudist-Walsh S, Murray RM, Nosarti C (2017): Real-life impact of executive function impairments in adults who were born very preterm. $J$ Int Neuropsychol Soc. 23: 381-389.

45. Nosarti C, Giouroukou E, Healy E, Rifkin L, Walshe M, Reichenberg A, et al. (2008): Grey and white matter distribution in very preterm adolescents mediates neurodevelopmental outcome. Brain. 131: 205-217.

46. Her Majesty's Stationary Office H (1991): Office of Population Censuses and Surveys, Standard Occupational Classification. London, UK: HMSO.

47. Annett M (1970): A classification of hand preference by association analysis. Br J Psychol. 61: 303-321.

48. Wechsler D (1999): Wechsler Abbrevaited Scale of Intelligence. New York: Psychologycal Corporation.

49. Nosarti C, Walshe M, Rushe TM, Rifkin L, Wyatt J, Murray RM, Allin MP (2011): Neonatal ultrasound results following very preterm birth predict adolescent behavioral and cognitive outcome. Dev Neuropsychol. 36: 118-135. 
50. Dale AM, Fischl B, Sereno MI (1999): Cortical surface-based analysis. I. Segmentation and surface reconstruction. Neuroimage. 9: 179-194.

51. Fischl B, Sereno MI, Dale AM (1999): Cortical surface-based analysis. II: Inflation, flattening, and a surface-based coordinate system. Neuroimage. 9: 195-207.

52. Schaer M, Cuadra MBB, Schmansky N, Fischl B, Thiran J-P, Eliez S (2012): How to measure cortical folding from MR images: a step-by-step tutorial to compute local gyrification index. $J$ Vis Exp. Jan 2: e3417.

53. IBM Corporation (2016): IBM SPSS Statistics for Windows, version 24.0.

54. Nosarti C, Al-Asady MHS, Frangou S, Stewart AL, Rifkin L, Murray RM (2002): Adolescents who were born very preterm have decreased brain volumes. Brain. 125: 1616-23.

55. Gautam P, Anstey KJ, Wen W, Sachdev PS, Cherbuin N (2015): Cortical gyrification and its relationships with cortical volume, cortical thickness, and cognitive performance in healthy mid-life adults. Behav Brain Res. 287: 331-339.

56. Spinillo A, Montanari L, Gardella B, Roccio M, Stronati M, Fazzi E (2009): Infant sex, obstetric risk factors, and 2-year neurodevelopmental outcome among preterm infants. Dev Med Child Neurol. 51: 518-25.

57. Kent AL, Wright IMR, Abdel-Latif ME, New South Wales and Australian Capital Territory Neonatal Intensive Care Units Audit Group (2012): Mortality and adverse neurologic outcomes are greater in preterm male infants. Pediatrics. 129: 124-31.

58. Platt MJ, Cans C, Johnson A, Surman G, Topp M, Torrioli MG, Krageloh-Mann I (2007): Trends in cerebral palsy among infants of very low birthweight $(<1500 \mathrm{~g})$ or born prematurely (<32 weeks) in 16 European centres: a database study. Lancet. 369: 43-50.

59. Benavides A, Metzger A, Tereshchenko A, Conrad A, Bell EF, Spencer J, et al. (2019): Sexspecific alterations in preterm brain. Pediatr Res. 85: 55-62.

60. Kozhemiako N, Nunes AS, Vakorin VA, Chau CMY, Moiseev A, Ribary U, et al. (2019): Sex differences in brain connectivity and male vulnerability in very preterm children. Hum Brain Mapp. 1-13.

61. Vasileiadis GT, Thompson RT, Han VKM, Gelman N (2009): Females follow a more "compact" early human brain development model than males. A case-control study of preterm neonates. Pediatr Res. 66: 551-554.

62. Kaczkurkin AN, Raznahan A, Satterthwaite TD (2019): Sex differences in the developing brain: insights from multimodal neuroimaging. Neuropsychopharmacology. 44: 71-85.

63. Shaw P, Kabani NJ, Lerch JP, Eckstrand K, Lenroot R, Gogtay N, et al. (2008): Neurodevelopmental trajectories of the human cerebral cortex. J Neurosci. 28: 3586-3594.

64. Cao B, Mwangi B, Passos IC, Wu M-J, Keser Z, Zunta-Soares GB, et al. (2017): Lifespan gyrification trajectories of human brain in healthy individuals and patients with major psychiatric disorders. Sci Rep. 7: 511.

65. Afif A, Bouvier R, Buenerd A, Trouillas J, Mertens P (2007): Development of the human fetal insular cortex: study of the gyration from 13 to 28 gestational weeks. Brain Struct Funct. 212: 335-346.

66. Wai MSM, Shi C, Kwong WH, Zhang L, Lam WP, Yew DT (2008): Development of the human insular cortex: differentiation, proliferation, cell death, and appearance of 5HT-2A receptors. Histochem Cell Biol. 130: 1199-1204.

67. Palaniyappan L, Crow TJ, Hough M, Voets NL, Liddle PF, James S, et al. (2013): Gyrification 
of Broca's region is anomalously lateralized at onset of schizophrenia in adolescence and regresses at 2 year follow-up. Schizophr Res. 147: 39-45.

68. Silani G, Lamm C, Ruff CC, Singer T (2013): Right supramarginal gyrus is crucial to overcome emotional egocentricity bias in social judgments. J Neurosci. 33: 15466-15476.

69. Cattaneo L, Rizzolatti G (2009): The mirror neuron system. Arch Neurol. 66: 557-560.

70. Mesulam MM (1998): From sensation to cognition. Brain. 121: 1013-1052.

71. Olausson H, Lamarre Y, Backlund H, Morin C, Wallin BG, Starck G, et al. (2002): Unmyelinated tactile afferents signal touch and project to insular cortex. Nat Neurosci. 5: 900904.

72. Shinder ME, Newlands SD (2014): Sensory convergence in the parieto-insular vestibular cortex. J Neurophysiol. 111: 2445-2464.

73. Craig ADB (2009): How do you feel--now? The anterior insula and human awareness. Nat Rev Neurosci. 10: 59-70.

74. Castagnini AC, Foldager L, Caffo E, Thomsen PH (2016): Early-adult outcome of child and adolescent mental disorders as evidenced by a national-based case register survey. Eur Psychiatry. 38: 45-50.

75. Copeland WE, Wolke D, Shanahan L, Costello J (2015): Adult functional outcomes of common childhood psychiatric problems. A prospective, longitudinal study. JAMA Psychiatry. 72: 892899.

76. Wolke D, Waylen A, Samara M, Steer C, Goodman R, Ford T, Lamberts K (2009): Selective drop-out in longitudinal studies and non-biased prediction of behaviour disorders. $\mathrm{Br} J$ Psychiatry. 195: 249-256.

77. Greene DJ, Black KJ, Schlaggar BL (2016): Considerations for MRI study design and implementation in pediatric and clinical populations. Dev Cogn Neurosci. 18: 101-112.

78. Luyt K, Jary S, Lea C, Young GJ, Odd D, Miller H, et al. (2019): Ten-year follow-up of a randomised trial of drainage, irrigation and fibrinolytic therapy (DRIFT) in infants with posthaemorrhagic ventricular dilatation. Health Technol Assess (Rockv). (Vol. 23). doi: 10.3310/hta23040.

79. Cheong JLY, Anderson PJ, Burnett AC, Roberts G, Davis N, Hickey L, et al. (2017): Changing neurodevelopment at 8 years in children born extremely preterm since the 1990s. Pediatrics. 139: e20164086.

80. Van Lieshout RJ, Boyle MH, Saigal S, Morrison K, Schmidt LA (2015): Mental health of extremely low birth weight survivors in their 30s. Pediatrics. 135: 452-459.

81. Pyhälä R, Wolford E, Kautiainen H, Andersson S, Bartmann P, Baumann N, et al. (2017): Selfreported mental health problems among adults born preterm: a meta-analysis. Pediatrics. 139: e201626 